\title{
Moyens aéroportés en télédétection
}

\section{Airborne teledetection equipment}

\author{
Alain Baudoin \\ Ingénieur Géographe \\ Institut Géographique National
}

\section{Introduction}

A l'ère spatiale les espoirs de la télédétection se trouvent naturellement vers les capteurs embarqués à bord des satellites d'observation de la terre. La NASA vient de lancer son troisième satellite Landsat et la France prépare son premier Spot pour 1983. Cependant si les missions spatiales forment l'une des composantes de la télédétection, elles n'en représentent qu'un aspect.

La télédétection est née avec la photo interprétation par l'utilisation de chambres photographiques embarquées à bord de ballons (Nadar en 1855), puis d'avions.

Elle s'est développée en utilisant d'autres capteurs, d'autres vecteurs et en mettant au point des méthodes nouvelles d'exploitation des données.

Nous allons exposer ici les différentes possibilités offertes par la télédétection aéroportée et décrire les moyens qu'elle met en ouvre.

\section{La photo interprétation}

La plus ancienne des techniques de télédétection est l'interprétation des photographies aériennes avec la seule aide d'un stéréoscope.

La photo interprétation reste dans de nombreux domaines une technique simple et relativement peu couteuse.

\section{Les avions}

Les véhicules utilisés pour effectuer les prises de vues photographiques sont le plus souvent des avions qui doivent être adaptés aux types de missions à réaliser.

L'Institut Géographique National possède par exemple les 4 types d'appareils suivants :

\section{- Biréacteur Mystère 20 - Fan Jet Falcon}

Avion moderne à hautes performances dont l'autonomie a été accrue par l'adjonction de réservoirs supplémentaires

Quelques caractéristiques

Charge utile avec plein de carburant

et équipage. . . . . . . . . . $0,400 \mathrm{t}$

Consommation horaire moyenne ... 900 \&

Autonomie.............. 5 5 H $45 \mathrm{~m}$

Distance maximale franchissable. . . $4250 \mathrm{Km}$

Vitesse de croisière . . . . . . . . $800 \mathrm{Km} / \mathrm{H}$

Plafond de travail .......... $12500 \mathrm{~m}$

\section{Equipage}

-1 pilote

- 1 navigateur situé au poste de copilote

- les opérateurs nécessaires à la mise en ouvre des appareillages embarqués

\section{Missions}

- photographies verticales : 2 trappes de prise de vues permettent de prendre des clichés simultanément sur deux émulsions avec des chambres RC 8 ou RC9 ainsi qu'avec des chambres RC 10 télécommandées du poste navigateur par l'intermédiaire d'un viseur Wild NF 2

- missions scientifiques diverses : infrarouge spatial (l'avion peut recevoir un télescope), étalonnage de moyens de guidage et de repérage, etc.

\section{Equipement}

- avion équipé pour le vol aux instruments

- radar météo

- navigateur Doppler

- viseur de navigation périscopique Wild NF2. 


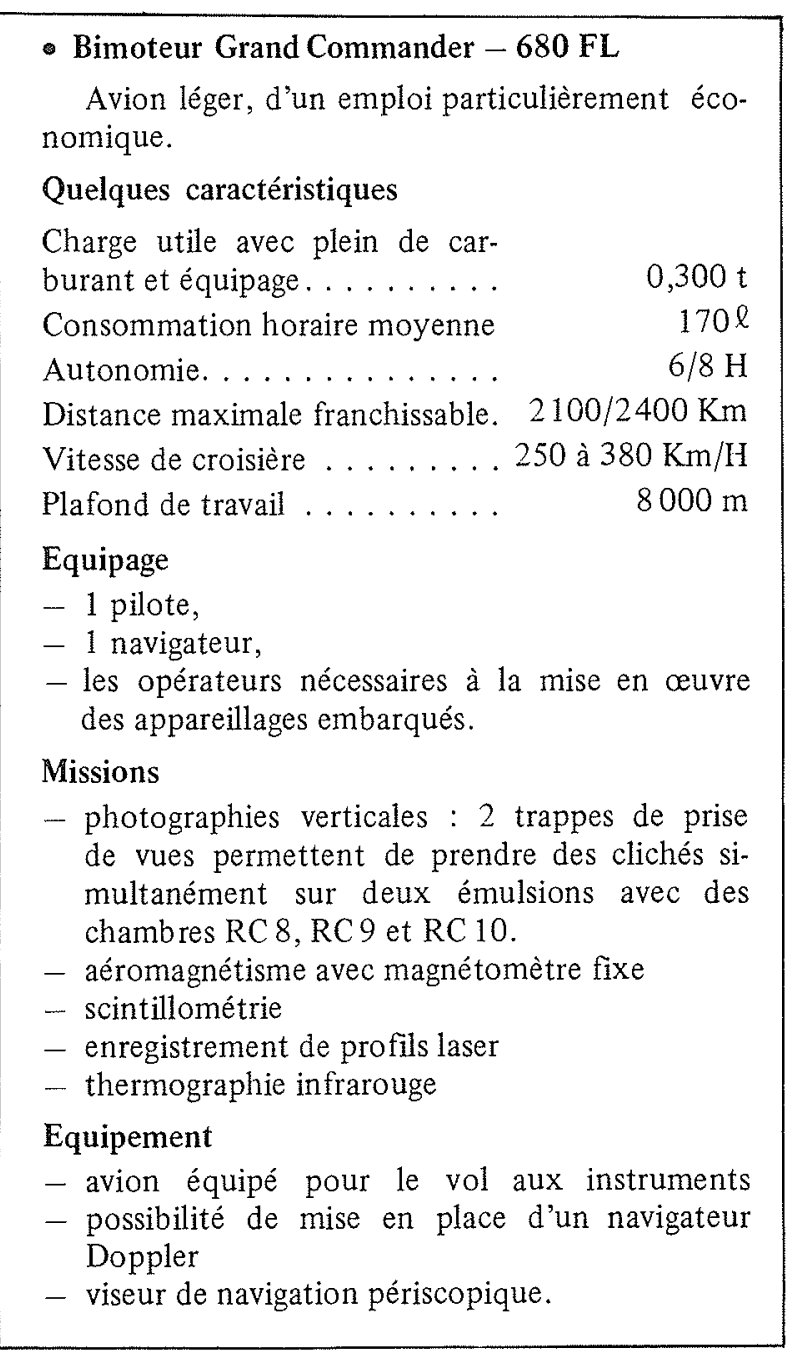

\section{- Bimoteur Hurel Dubois - HD 34}

Avion à décollage court, doté d'une grande autonomie et susceptible d'opérer à très faible vitesse.

\section{Quelques caractéristiques}

Charge utile avec plein de car-

burant et équipage. . . . . . . . .

Consommation horaire moyenne

Autonomie. ...........

Distance maximale franchissable

$3600 \mathrm{Km}$

Vitesse de croisière . . . . . 200 à $280 \mathrm{Km} / \mathrm{H}$

Plafond de travail ........ $6000 \mathrm{~m}$

Equipage

-1 pilote

- 1 navigateur

- 1 copilote ou mécanicien

- les opérateurs nécessaires à la mise en cuvre des appareillages embarqués.

\section{Missions}

- photographies verticales : 2 trappes de prise de vues permettant de prendre des clichés simultanément sur deux émulsions avec des chambres RC 8, RC 9 ou RC 10
- photographies obliques par deux trappes latérales

- aéromagnétisme avec magnétomètre tracté

- scintillométrie

- enregistrements de profils par APR ou laser

- thermographie infrarouge etc.

Equipement

- avion équipé pour le vol aux instruments

- navigateur Doppler

- viseur de navigation périscopique.

\section{- Quadrimoteur B. 17}

Bombardier de la dernière guerre mondiale cet avion aux qualités exceptionnelles a pu être conservé en parfait égat de vol grace aux stocks de rechanges dont dispose le S.A.A.

\section{Quelques caractéristiques}

Charge utile avec plein de car-

burant et équipage. . . . . . . . .

Consommation horaire moyenne

Autonomie. . . . . . . . . .

$11 / 15 \mathrm{H}$

Distance maximale franchissable

$4000 \mathrm{Km}$

Vitesse de croisière . . . . . . 250 à $380 \mathrm{Km} / \mathrm{H}$

Plafond de travail ........ $8500 \mathrm{~m}$

Equipage

-1 pilote

- 1 copilote ou mécanicien

- 1 navigateur et les opérateurs nécessaires à la mise en oeuvre des appareillages embarqués.

\section{Missions}

- photographies verticales : plusieurs trappes de prise de vues permettent de prendre des clichés simultanément sur plusieurs émulsions avec des chambres RC 8, RC 9 ou RC 10

- photographies obliques

- aéromagnétisme avec magnétomètre tracté

- enregistrement de profils par APR ou laser

- thermographie infrarouge

- missions scientifiques diverses : étalonnage de stations de poursuite de satellites, etc.

\section{Equipement}

- avion équipé pour le vol aux instruments

- navigateur Doppler

- viseur de navigation périscopique

- possibilité de montage de chaînes de navigation Shoran, Loran, Decca, Toran, etc.

Les chambres photographiques

Les chambres métriques utilisées en photogrammétrie sont également employées en télédétection.

Par exemple les chambres suivantes de l'I.G.N. (matériel de prise de vues)

\section{Les films}

Avec les chambres photographiques, des images de la surface du sol à différentes échelles peuvent être 


\begin{tabular}{|c|c|c|c|c|c|c|c|}
\hline \multicolumn{8}{|c|}{ Matériel de prise de vues } \\
\hline Nombre & Constructeur & Type & Support & Objectif & $\begin{array}{l}\text { Focale } \\
\text { en } \mathrm{mm}\end{array}$ & $\begin{array}{l}\text { Format } \\
\text { en } \mathrm{cm}\end{array}$ & Emulsions utilisées \\
\hline 2 & Wild & RC 8 & $\begin{array}{l}\text { Film } \\
0,24 \times 60 \mathrm{~m}\end{array}$ & Aviogon & 152 & $23 \times 23$ & $\begin{array}{l}\text { Panchromatique, infra-rouge } \\
\text { avec filtre correcteur de } \\
\text { focale }\end{array}$ \\
\hline 7 & Wild & RC 8 & $\begin{array}{l}\text { Film } \\
0,24 \times 60 \mathrm{~m}\end{array}$ & $\begin{array}{l}\text { Universal } \\
\text { Aviogon }\end{array}$ & 152 & $23 \times 23$ & $\begin{array}{l}\text { Panchromatique, infra-rouge, } \\
\text { couleurs, fausses couleurs }\end{array}$ \\
\hline 4 & Wild & $R C 8$ & $\begin{array}{l}\text { Film } \\
0.19 \times 60 \mathrm{~m}\end{array}$ & Aviotar & 210 & $18 \times 18$ & $\begin{array}{l}\text { Panchromatique, infra-rouge } \\
\text { avec filtre correcteur de } \\
\text { focale, couleurs, fausses } \\
\text { couleurs }\end{array}$ \\
\hline 2 & Wild & RC 9 & $\begin{array}{l}\text { Film } \\
0,24 \times 60 \mathrm{~m}\end{array}$ & $\begin{array}{l}\text { Super } \\
\text { Aviogon }\end{array}$ & 88 & $23 \times 23$ & Panchromatique \\
\hline 1 & Wild & $R \subset 9$ & $\begin{array}{l}\text { Film } \\
0,24 \times 60 \mathrm{~m}\end{array}$ & $\begin{array}{l}\text { Super } \\
\text { Aviogon }\end{array}$ & 88 & $23 \times 23$ & Infra-rouge \\
\hline 2 & 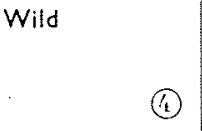 & $\mathrm{RC} 10$ & $\begin{array}{l}\text { Film } \\
0,24 \times 120 \mathrm{~m}\end{array}$ & $\begin{array}{l}\text { Universal } \\
\text { Aviogon } \\
\text { et Super } \\
\text { Aviogon } 11\end{array}$ & $\begin{array}{l}152 \\
\text { et } \\
88\end{array}$ & $23 \times 23$ & $\begin{array}{l}\text { Panchromatique, infra-rouge } \\
\text { couleurs, fausses couleurs }\end{array}$ \\
\hline 1 & $\begin{array}{l}\text { Zeiss } \\
\text { avec viseur (3) }\end{array}$ & RMK & $\begin{array}{l}\text { Film } \\
0.24 \times 120 \mathrm{~m}\end{array}$ & Telikon & 610 & $23 \times 23$ & $\begin{array}{l}\text { Panchromatique, infra-rouge, } \\
\text { couleurs, fausses couleurs }\end{array}$ \\
\hline
\end{tabular}

effectuées, pouvant aller du $1: 500$ au $1: 100000$ en fonction de la focale de l'objectif et de l'altitude de l'avion.

Les qualités de ces images dépendent essentiellement du type de film utilisé.

Les caractéristiques de celui-ci sont :

- sa résolution qui détermine la taille du plus petit objet décelable,

- sa ou ses réponses spectrales (selon qu'il est à une seule émulsion : blanc et noir, ou à trois émulsions : en couleurs),

Celles-ci déterminent le type de rayonnement (bande spectrale) analysé, qui peut-être modifié par l'emploi de filtres colorés ;

- sa rapidité qui détermine la quantité de lumière qui permet d'impressioner l'émulsion.

Les films les plus utilisés sont:

\section{Film panchromatique}

La sensibilité limitée au spectre visible (de 0,4 à 0.7 microns) de ce film et sa bonne résolution font qu'il est surtout utilisé en photogammétrie mais fournit des informations utiles en géologie, géomorphologie, urbanisme, aménagement etc.

\section{Film infra rouge (noir et blanc)}

Sa sensibilité se prolonge au-delà de 0,7 microns jusqu'au proche infra rouge $(0,9 \mu)$. Sa résolution est moins grande que celle du panchromatique mais il per- met de mettre en évidence toutes les eaux, absorbantes dans l'infrarouge, qui apparaissent en noir sur les images alors que la végétation chlorophylienne qui réfléchit bien ce rayonnement ressort en clair dans la photographie.

Cet avantage est exploité en hydrologie, en agriculture par exemple.

\section{Film en couleurs naturelles.}

Ses trois couches respectivement sensibles au bleu, vert et rouge lui permettent de reconstituer les couleurs perçues par l'œil humain. Cet avantage est surtout intéressant en pédologie, pour l'aménagement de l'espace, et dans l'étude des eaux littérales.

\section{Film en fausses couleurs (infra rouge couleurs)}

Ses trois couches sont sensibles au vert, au rouge et au proche infra rouge alors qu'elles sont colorées respectivement en bleu, vert et rouge si bien que. ce film allie les avantages du film infra rouge et du film couleurs : la végétation apparaît en rouge, l'eau en noir.

Ce film est employé pour les études d'occupation du sol, de la pollution etc.

\section{Les autres vecteurs}

L'avion est limité à une certaine gamme d'altitudes (de $500 \mathrm{~m}$ à $15000 \mathrm{~m}$ environ) et de vitesses (de 100 à $1000 \mathrm{~km} / \mathrm{h}$ ). 
Pour aller plus haut, sans utiliser les satellites, le ballon stratosphérique est intéressant. Il permet d'atteindre 30 à $50 \mathrm{~km}$. Cependant sa trajectoire, si elle peut être calculée, ne peut pas être choisie de façon quelconque par l'utilisateur : elle doit s'accorder de la direction du vent.

Les fusées sondes peuvent également être porteuses d'appareils photographiques. Comme dans le cas des ballons, ceux-ci sont souvent de petit format $(70 \mathrm{~mm})$.

L'utilisation d'un hélicoptère est très apprécié pour effectuer des missions à très grande échelle ou pour surveiller des zones évolutives (cas de pollutions de l'eau en particulier).

\section{Les prises de vues obliques}

Pour de nombreuses applications les prises de vues sont à axe vertical et avec un recouvrement qui permet leur exploitation stéréoscopique. Cependant, pour étudier certains phénomènes, les prises de vues obliques sont les plus intéressantes. C'est le cas de l'archéologie : les traces de vestiges enfouis apparaissent à la surface du sol dans certaines conditions particulières de sécheresse, d'éclairage par le soleil et d'inclinaison de la chambre photographique.

\section{La thermographie aéroportée}

Les capteurs non photographiques les plus utilisés sont les radiomètres à balayage qui analysent le terrain dans les bandes spectrales du rayonnement thermique du sol (de 3 à 15 microns).

Les images reconstituées sur un film sont des thermographies et révèlent le comportement thermique des objets qui dépend tout d'abord de leur température.

Les satellites météorologiques (NOAA, Metéosat etc) possèdent des capteurs infra rouge thermiques utilisés pour étudier les masses nuageuses, mais sont peu adaptés par leur mauvaise résolution aux études concernant la surface terrestre. Même le satellite Landsat 3 qui vient d'être mis sur orbite est équipé d'un radiomètre thermique ayant une résolution au sol de $250 \mathrm{~m}$.

\section{Les applications de la thermographie sont nombreuses}

- Recherches d'eau douce sur le littoral : les résurgences qui ont une température différente de celle de la mer sont visibles sur la thermographie, ce qui permet de les localiser de façon précise. Une enquête sur le terrain fournit les renseignements complémentaires pour éliminer les résurgences d'eau de mer et choisir les sources exploitables.

- En agriculture et dans l'étude de l'occupation des sols, la thermographie peut être utile pour fournir des informations complémentaires à celles des photographies aériennes sur l'état du sol et des cultures. Notons cependant les difficultés d'interprétation des mesures radiométriques qui ne foumissent pas directement la température du sol.

- En urbanisme et dans le domaine des économies d'énergie la thermographie peut être d'une aide précieuse pour ausculter l'isolation thermique des habitations et des bâtiments. Chaque fuite, le long des faîtières ou devant les ouvertures, est mise en évidence sur la thermographie.

- La thermographie est très bien adaptée à l'hydrologie, à la courantologie et à toutes les études concernant l'eau. Le rayonnement thermique de cette dernière ne dépend pratiquement que de sa température, sauf si celle ci est polluée par des hydrocarbures ou d'autres substances.

Aussi la thermographie est très utile pour suivre les échanges entre la mer et les étangs littoraux, pour surveiller le rejet des eaux d'une centrale thermique, pour localiser les rejets d'eaux polluées.

Dans toutes ces applications il est essentiel d'effectuer plusieurs prises de vues dans des conditions précises de vents, de courant, de marée, de température.

Aussi l'emploi des véhicules aéroportés est indispensable pour ces missions.

En outre les phénomènes à analyser sont souvent ponctuels ou situés dans une bande étroite de terrain (littoral ou rivière) : le vecteur le mieux adapté est alors l'hélicoptère qui permet des mesures à très basse altitude.

\section{Les mesures complémentaires}

Afin d'exploiter les mesures thermographiques, il est presque toujours indispensable de compléter ces informations par des mesures au sol. La température de l'eau est la plus importante, mais d'autres paramètres peuvent être enregistrés (humidité, température de l'air, vitesse et direction du vent etc.). Ces renseignements permettent de corriger ou d'étalonner les mesures aéroportées.

Mentionnons que pour obtenir des mesures concernant l'atmosphère, la Météorologie Nationale a mis au point des "avions modèle réduit" télécommandés et que pour des mesures sub-aquatiques des bouées immergées peuvent être utilisées.

\section{L'exploitation des données thermographiques}

Les mesures aéroportées, complétées par celles réalisées au sol, permettent la réalisation de différents types de documents cartographiques:

- Les isothermes peuvent être mises en évidence soit par des méthodes d'équidensités, soit par des traitements numériques qui offrent la possibilité de corriger radiométriquement et géométriquement les données.

- La cartographie des rejets fournit la localisation de ceux-ci sur le rivage et une indication de leur importance, en matérialisant les fronts thermiques qu'ils produisent.

\section{Les autres capteurs aéroportés}

A cette panoplie du télédétecteur d'autres capteurs peuvent être ajoutés : 


\section{Les capteurs multispectraux}

- Des chambres photographiques ayant plusieurs objectifs (4 à 9) sont employées pour enregistrer sur le même film, à l'aide de plusieurs filtres, les images dans différentes bandes spectrales de la même scène. Ces images "noir et blanc" sont ensuite exploitées en recréant des images colorées à l'aide de trois d'entre elles projetées sur un même écran de visualisation avec trois sources lumineuses de couleurs bleue, verte et rouge.

- Les systèmes à balayage multispectraux permettent une analyse plus fine des bandes spectrales du rayonnement visibles. Certains d'entre eux ont jusqu'à 20 canaux qui fournissent autant d'images différentes du terrain.

De tels capteurs, qui sont bien adaptés à l'utilisation spatiale sont cependant intéressant pour des expérimentations aéroportées. Ils permettent d'évaluer l'intérêt de telle ou telle bande spectrale pour telle ou telle utilisation, ainsi que les conditions optimales de leur emploi (époque de prise de vue, heure par exemple).

De plus ces capteurs fournissent des images qui peuvent simuler celles qui seront produites par les satellites futurs et servent de banc d'essai pour mettre au point les méthodes de traitement de ces images.

\section{Les radars latéraux}

L'inconvénient des chambres photographiques et des capteurs à balayage est de ne pouvoir obtenir d'images du terrain que par beau temps en l'absence de nuages. Cette contrainte peut être très génante dans certains pays à forte nebulosité (Brésil, Gabon etc). Dans ce cas l'utilisation d'un radar permet de résoudre en partie le problème. Le rayonnement enregistré par un tel capteur a une longueur d'onde très grande par rapport à celle du rayonnement visible (quelques centimètres au lieu de 0,5 microns). Ces micro-ondes ont la propriété de ne pas être absorbées par les nuages ce qui permet au radar d'explorer le sol par tous les temps.
Le radar utilisé sur un avion est appelé radar latéral car il analyse le paysage, non pas en dessous de lui, mais sur le côté

Un tel capteur est adapté à la cartographie à petite échelle $(1: 100000)$ des pays tropicaux humides, à la prospection géologique, à la reconnaissance des routes des navires au milieu des glaces. Cet instrument encore très couteux est sûrement appeler à se développer rapidement.

\section{Capteurs divers}

Signlons rapidement des instruments qui peuvent être embarqués à bord d'un avion pour réaliser des missions particulières de télédétection :

- Les radiomètres à micro-ondes qui analysent le rayonnement émis par le sol dans les longueurs d'ondes du radar (quelques $\mathrm{mm}$ à quelques $\mathrm{dm}$ ).

- Les lasers à fluorescence qui étudient l'émission particulière d'une lumière par certains corps chimiques lorsqu'ils sont éclairés. (Ces capteurs peuvent être intéressant pour la recherche minière).

\section{Conclusion}

La grande souplesse offerte par les moyens aéroportés en matière de télédétection donne à ceux-ci un intérêt très grand pour de nombreuses disciplines où le point de vue du satellite est trop élevé et celui de l'observateur au sol trop bas.

Dans les années à venir le rôle de l'avion restera très important, en particulier pour la réalisation de prise de vues photographiques à grande échelle, mais se modifiera sans doute pour devenir le complément indispensable du satellite en permettant à ce dernier d'extrapoler sur des surfaces très grandes les mesures précises obtenues par voie aérienne et par sondage sur le terrain.

\section{Discussion}

Le Président remercie M. OSCHE, représentant M. Baudoin, de son exposé et il ouvre la discussion en échangeant avec lui quelques réflexions sur la complémentarité des champs d'application de l'avion ou du satellite.

M. OSCHE observe notamment que, loin de se faire concurrence sur un même domaine, satellites et moyens aéroportés laissent subsister des plages intermédiaires impliquant l'intervention d'autres procédures (fusées sondes, ballons) ou -mieux encore - l'amélioration des capteurs embarqués.

Une série de questions posées par $\mathrm{M}$. COROMPT amène
M. OSCHE à préciser la nature des équipements mis en œuvre et des renseignements recueillis selon les bandes spectrales explorées.

Le visible classique et l'infrarouge proche -jusqu'à 0,9 micron- est du domaine de la photographie. Au-dejà et en particulier pour les deux "fenêtres" ouvertes dans l'écran atmosphérique entre 3 et 5 microns et entre 8 et 14 microns, l'information est saisie par le capteur à balayage et enregistrée soit en mode analogique, soit en mode numérique. La restitution des enregistrements peut prendre la forme et donne d'ailleurs l'illusion de photographies. 